\title{
A FM quadrature demodulation method based on small angle approximation
}

\author{
Yang Yang ${ }^{1, a}$, Li Xueyuan ${ }^{1, b}$, Cheng Nannan ${ }^{1, c}$, Huang Dongdong ${ }^{1, d}$ \\ ${ }^{1}$ North China University of Technology Beijing, China \\ aYangY@ncut.edu.cn, ${ }^{b}$ lixueyuan0101@163.com, cheng_nn@ncut.edu.cn \\ dhuang_dong_dong@yeah.net
}

Keywords: FM demodulation; small angle approximation; software radio

\begin{abstract}
This paper introduces a FM quadrature demodulation method based on small angle approximation; this method can complete the task of FM signal demodulation using all digital circuit. After setting up the FM demodulation hardware test platform based on the software radio, the FM demodulation method is tested. The result shows that the method can demodulate FM signal reliable, and have higher efficiency than traditional method.
\end{abstract}

\section{Introduction}

The digital receiver system based on software defined radio (SDR) has received great attention and extensive interest [1]. SDR requires AD and DA converter closes to the antenna as much as possible to realize the wireless communication with the modern software technology, which is achieved by hardware method traditionally [2]. The SDR has strong flexibility, the structure, protocol and standard of communication system could be updated and modified by the upgrade and expansion of software model [3]. The compatibility of equipment will be improved and the service life will be prolonged.

At present, the traditionally analog communication system is being gradually replaced by digital system, as one of the most widely used analog modulation application, frequency modulation (FM), has been widely used in radio, television and cellular telephone system. Currently, FM continues to be used in stereo broadcast, trucking communication, radio etc. In order to ensure that the system is compatible with the original one, SDR can be used to make FM demodulation module embedded into digital system. The new system could be expanded with compatibility of old system. Under this background, this paper puts forward a kind of a FM quadrature demodulation method based on small angle approximation using SDR. This system could receive FM broadcast signal from the antenna directly, and complete the FM demodulation in all digital domain.

\section{Basic FM digital demodulation method}

Digital phase locked loop (PLL) and quadrature demodulation are commonly used digital demodulation method for FM signals. Digital PLL is composed of a phase detector (PD), loop filter (LF) and numerically controlled oscillator (NCO) [4]. After the RF signal is received by the analog front end, $A D$ convert will send the output to the $\mathrm{PD}$, which will be compared with NCO output. LF will process the result from PD after filter the high frequency components; Then NCO accepts the difference frequency components as input to control the output frequency [5]. The output of NCO will be regulated according to PD, so as to realize the locking of the input signal frequency, complete signal demodulation (Figure 1).

Another method is using digital quadrature demodulation, as shown in Figure 2, after quadrature demodulation of FM signal, the in-phase components $I(n)$ and quadrature components $Q(n)$ is separated through the low pass filter, demodulated baseband signal could be obtained by an arctan operation [6].

Using the above digital demodulation method will lead the following problems:

The problem of carrier synchronization. PLL method need adjust the parameters of LF and NCO 
to ensure output carrier frequency of NCO not differs too much as the FM carrier frequency, which will cause PLL unable to lock. The method of quadrature demodulation needs coherent carrier consistent with FM carrier frequency, carrier synchronization is also needed.

The problem of computational complexity. Orthogonal demodulation method requires a division, an arctan and a subtraction operation, arctan calculations generally use the CORDIC algorithm [7]. The disadvantage of this method is the complexity of the system, operation speed and precision is inversely affected, there is no way to simultaneously ensure the speed and precision.

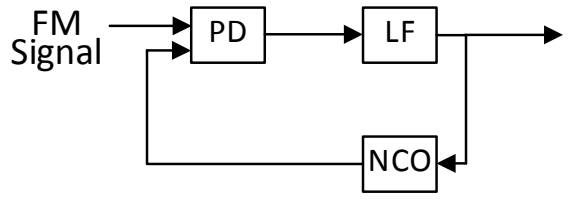

Figure 1 PLL Demodulation Method

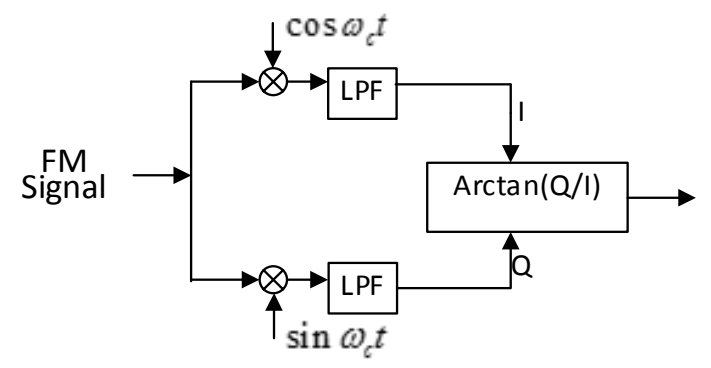

Figure 2 Orthogonal Demodulation Method

Based on the above reasons, this paper puts forward an orthogonal demodulation method based on small angle approximation. This method does not require carrier synchronization, need simple operation and easy to realize.

\section{Improved FM digital demodulation method}

According to the basic principle of angle modulation, we can express FM signal as:

$S_{0}(t)=A \cos \left[\omega_{c} t+\varphi(t)\right]$

Where $\mathrm{A}$ is the amplitude of carrier frequency, $\left[\omega_{c} t+\varphi(t)\right]$ is the signal Instantaneous Phase, $\varphi(t)$ is the instantaneous phase shift of $\omega_{c} t$.

Local carrier is generated as:

$\cos \left(\omega_{c} t+\Delta \omega t\right)$

$\sin \left(\omega_{c} t+\Delta \omega t\right)$

Where $\omega_{c}$ is same frequency carrier, $\Delta \omega$ is an offset.

Multiply frequency modulation signal with the local generated orthogonal carrier is shown as:

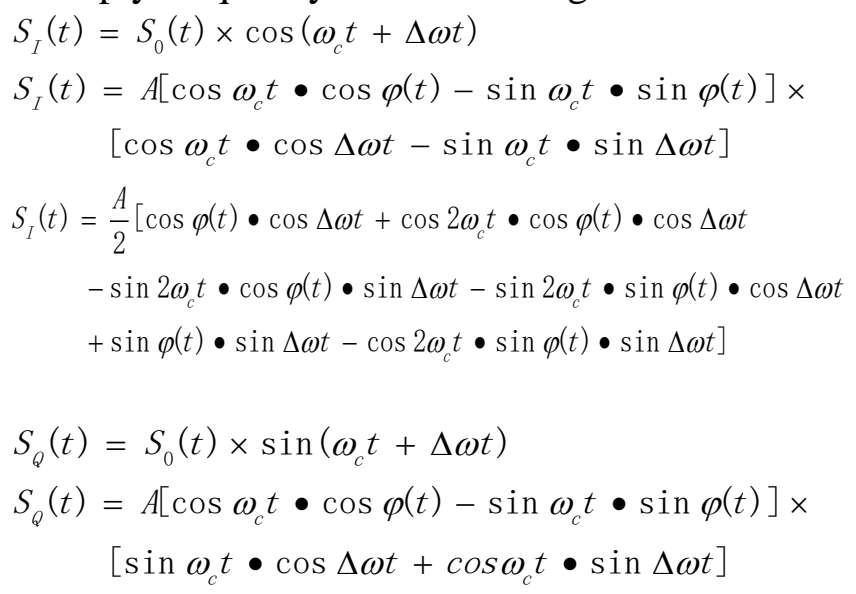




$$
\begin{aligned}
S_{\theta}(t) & =\frac{A}{2}\left[\sin 2 \omega_{c} t \bullet \cos \varphi(t) \bullet \cos \Delta \omega t+\cos \varphi(t) \bullet \sin \Delta \omega t\right. \\
& +\cos 2 \omega_{c} t \bullet \cos \varphi(t) \bullet \sin \Delta \omega t-\sin \varphi(t) \bullet \cos \Delta \omega t \\
& \left.+\cos 2 \omega_{c} t \bullet \sin \varphi(t) \bullet \cos \Delta \omega t-\sin 2 \omega_{c} t \bullet \sin \varphi(t) \bullet \sin \Delta \omega t\right]
\end{aligned}
$$

Filter $S_{I}(t)$ and $S_{Q}(t)$ with a low pass filter, the high frequency component with $2 \omega t$ :

$I(t)=\frac{A}{2}[\cos \varphi(t) \bullet \cos \Delta \omega t+\sin \varphi(t) \bullet \sin \Delta \omega t]$

$Q(t)=\frac{A}{2}[\cos \varphi(t) \bullet \sin \Delta \omega t-\sin \varphi(t) \bullet \cos \Delta \omega t]$

When angle is small, we can consider $\sin \varphi(t) \approx \varphi(t), \cos \varphi(t) \approx 1$, so

$$
\begin{aligned}
I(t) & =\frac{A}{2}[\cos \varphi(t) \bullet \cos \Delta \omega t+\varphi(t) \bullet \sin \Delta \omega t] \\
Q(t) & =\frac{A}{2}[\cos \varphi(t) \bullet \sin \Delta \omega t-\varphi(t) \bullet \cos \Delta \omega t]
\end{aligned}
$$

The sum of square of equation (1) and equation (2) is shown as:

$$
I^{2}(t)+Q^{2}(t)=\varphi^{2}(t)+1
$$

We can see from the above process, the modulating signal could be recovered. And the frequency offset of carrier synchronization does not need to be considered. At the same time, the operation is simple and easy to realize.

\section{Realization of FM digital receiver}

According to the principles of the third part, we design a digital FM receiver based on FPGA. The receiver use standard SDR scheme, system consists of three parts, antenna, high speed AD converter and FPGA. We use a half meter copper cable as antenna, which connect to the AD converter through a SMA interface. The FM broadcast signal will be send to the high speed AD converter after it is received from antenna. System will demodulate the digital signal inside FPGA. AD converter use $24 \mathrm{M}$ clock, directly sample the FM signal of $100 \mathrm{MHz}$. According to the band pass sampling theorem, the signal will produce a mirror spectrum at $4 \mathrm{MHz}$. We use $4 \mathrm{MHz} \pm$ $500 \mathrm{KHz}$ band pass digital filter to filter out the signal. Then $4 \mathrm{MHz}$ orthogonal signal is used for demodulation, after passing through a low pass filter, and make a sum of square operation, we can recover the original modulated signal. The demodulation principle block diagram is showed in figure 3:

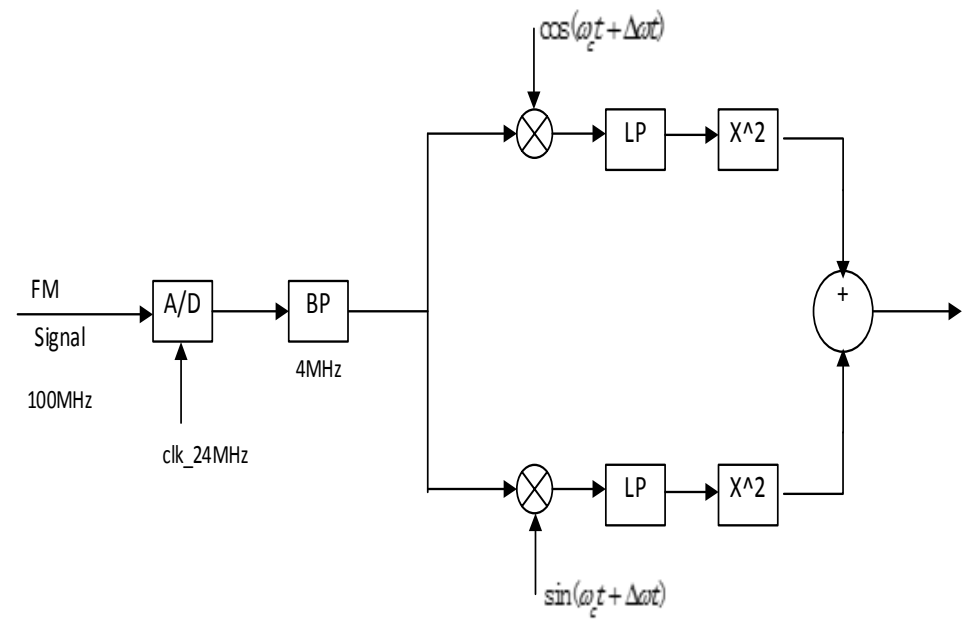

Figure 3 demodulation principle block diagram 
The hardware of test system is shown in figure 4, the system is divided into 4 parts:

1. Antenna, a copper wire of half a meter

2. AD converter, TI's 125M AD converter ADS62P15

3. FPGA, Xilinx's XC7A200T

4. Audio output, CIRRUS stereo D/A Converter CS4344

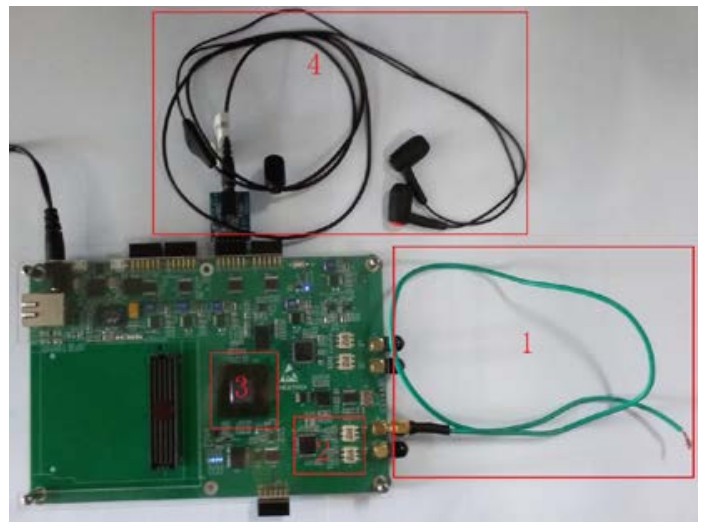

Figure 4 Hardware test Platform

The software of the whole system is implemented in FPGA, we use System Generator, which is a design tool for DSP Applications provided by Xilinx, realizes demodulation algorithm. After simulation, the NCO file is generated and added into the system project, and completes the digital FM receiver system. The finally experimental results show that the system can hear the FM broadcast clearly, and the primarily consumed resource is of less than $10 \%$.

\section{Conclusion}

This paper analyzes and compares the advantages and disadvantages of PLL demodulation and quadrature demodulation, puts forward a FM quadrature demodulation method based on small angle approximation. This method can be used for all digital demodulation for FM signal. After the introduction of basic principle, we build a FM demodulation hardware test platform based on SDR. The RF signal, what the antenna receives, will be converted to digital signal directly. All the demodulation process will be completed inside FPGA. Experimental results show that, the method can demodulate FM signal reliably and efficiently.;

\section{References}

[1] M.L. Dickens, B.P. Dunn and J.N. Laneman, :Design and Implementation of a Portable Software Radio,IEE Communications Magazine, vol. 46,no. 8(2008), pp. 58-66.

[2] Jeffrey H. Reed: Software Radio: A Modern Approach to Radio Engineering, Prentice Hall PTR, pp.540-550.

[3] I. Hatai and I. Chakrabarti:FPGA implementation of a digital FM modem for SDR architecture, Computers and Devices for Communication(2009),pp. 1-4,

[4] S.R. Schnelle, J.P. Slavinsky, P.T. Boufounos, M.A. Davenport and R.G. Baraniuk:A COMPRESSIVE PHASE-LOCKED LOOP, Acoustics, Speech and Signal Processing (ICASSP) (2012), pp.2885-2888.

[5] F. Gardner:Phaselock Techniques, Wiley-Interscience( 2005)

[6] S. Sunny;J.V. John and T.J. Apen:Quadrature detection methods for FM demodulation Advances in Computing and Communications (ICACC) (2013),pp.433-436.

[7] J. Valls.,T. Sansaloni ; A. Perez-Pascual , V.Torres and V. Almenar:The use of CORDIC in software defined radios: a tutorial, Computers and Devices for Communication (2009), pp.1-4. 